\title{
Optical frequency standard based on a single ${ }^{40} \mathrm{Ca}^{+}$
}

\author{
GAO KeLin \\ State Key Laboratory of Magnetic Resonance and Atomic and Molecular Physics, Key Laboratory of Atomic Frequency Standards, \\ Wuhan Institute of Physics and Mathematics, Chinese Academy of Sciences, Wuhan 430071, China
}

Received July 23, 2012; accepted September 10, 2012

\begin{abstract}
Research on the development of the optical frequency standard based on trapped and cold ${ }^{40} \mathrm{Ca}^{+}$with the $4 \mathrm{~s}{ }^{2} \mathrm{~S}_{1 / 2}-3 \mathrm{~d}^{2} \mathrm{D}_{5 / 2}$ clock transition at $729 \mathrm{~nm}$ is reported. A single calcium ion was trapped and laser cooled in the Paul trap and stay in trap for more than 15 days. The linewidth of a $729 \mathrm{~nm}$ laser was reduced to less than $10 \mathrm{~Hz}$ by locking to a cavity for longer than 50 hours uninterruptedly. The overall systematic uncertainty of the clock transition has been characterized to be better than $6.5 \times 10^{-16}$. The absolute frequency of the clock transition was measured at $10^{-15}$ level using an optical frequency comb referenced to a Hydrogen maser, which was calibrated to the SI second through the global positioning system (GPS). The frequency value was 411042129776393.0(1.6) Hz after the correction of the systematic shifts.
\end{abstract}

optical frequency standard, single calcium ion, systematic uncertainty, absolute frequency measurement

Citation: $\quad$ Gao K L. Optical frequency standard based on a single ${ }^{40} \mathrm{Ca}^{+}$. Chin Sci Bull, 2013, 58: 853-863, doi: 10.1007/s11434-012-5646-5

Accurate time and frequency standards have many applications such as the realization of the SI base units of time, satellite-based navigation, and tests of physical theories. Since 1967, the definition of the SI second is based on the ground hyperfine transition of the ${ }^{133} \mathrm{Cs}$ atom. Currently the Cs fountain primary standard with the smallest uncertainty ever reported has achieved the $10^{-16}$ level [1]. With high- $Q$ transitions, the optical frequency standards based on lasercooled trapped ions or atoms can achieve even better stability and accuracy. Optical frequency standards based on ultracold neutral atoms and single ions have been developed rapidly in recent years, such as ${ }^{87} \mathrm{Sr},{ }^{88} \mathrm{Sr},{ }^{171} \mathrm{Yb},{ }^{174} \mathrm{Yb},{ }^{88} \mathrm{Sr}^{+}$, ${ }^{171} \mathrm{Yb}^{+},{ }^{27} \mathrm{Al}^{+},{ }^{40} \mathrm{Ca}^{+}$and ${ }^{199} \mathrm{Hg}^{+}$[2-10]. Uncertainties on the order of $10^{-17}$ or even smaller were reported with $\mathrm{Al}^{+}$and $\mathrm{Hg}^{+}$[8]. The best evaluation of the frequency uncertainty reported so far was $8.6 \times 10^{-18}$ with $\mathrm{Al}^{+}$[11]. The uncertainties evaluated above, both based on single trapped ions or ultracold neutral atoms, have already surpassed those of the best Cs fountain clocks. They are expected to replace the Cs primary microwave standard for the definition of the SI second in the near future. Lasers (optical local oscillators)

email: klgao@wipm.ac.cn frequency stabilized to the transitions of atoms, ions, and molecules were recommended by the International Committee for Weights and Measures (CIPM) as secondary representations of the SI second, contributing to International Atomic Time (TAI) [12].

In China, there will be some optical frequency standards, such as $\mathrm{Sr}, \mathrm{Yb}, \mathrm{Ca}, \mathrm{Hg}, \mathrm{Al}^{+}, \mathrm{Ca}^{+}, \mathrm{Hg}^{+}, \mathrm{In}^{+}$and $\mathrm{Ba}^{+}$are being developed. And there will be some suggestion on the active optical clock [13].

The optical frequency standard based on ${ }^{40} \mathrm{Ca}^{+}$, which has been proposed as one of the candidates for the next definition of the SI second [12], are also being developed by the Quantum Optics and Spectroscopy Group in Innsbruck [9], the National Institute of Information and Communications (NICT) in Japan [14] and the Physique des Interactions Ioniques et Molecularies in France [15]. The technological advantage is that all necessary lasers could be generated by commercially available and easy-to-handle solid state lasers. And the odd isotope of ${ }^{43} \mathrm{Ca}^{+}$ion could be used as an optical frequency standard which is immune to the first-order Zeeman frequency shift. The ${ }^{40} \mathrm{Ca}^{+}$is also popular in atomic physics and quantum information.

In this paper, we report the experimental work on the 
development of the optical frequency standard based on a single ${ }^{40} \mathrm{Ca}^{+}$. First the experimental setup is described, including ion trap and laser systems. Then, the experiment results are represented, including experiments on single trapped and cooled $\mathrm{Ca}^{+}$, locking the optical frequency on $729 \mathrm{~nm}$ laser to the $\mathrm{Ca}^{+}$, characterizing the systematic shifts and uncertainties of the clock transition and measuring the absolute frequency of the clock transition using an optical frequency comb referenced to a hydrogen maser, which was calibrated to the SI second through the global positioning system (GPS). Last, we give the future prospect.

\section{Experimental setup}

To build an optical frequency standard, normally we need a frequency reference (the optical transitions of the ions or atoms), a local oscillator (the ultra narrow linewidth probe laser), and an apparatus transferring the oscillations from optical frequency region into the microwave region to realize the daily application of the frequency standard (the optical frequency comb). The overall schematic diagram of the experimental setup is shown in Figure 1. In the following parts, we will describe the experimental setup of our optical frequency standard, which can be separated mainly into three parts.

A partial energy level diagram of the ${ }^{40} \mathrm{Ca}^{+}$optical frequency standard is shown in Figure 2. The clock transition is the electric quadrupole transition $4 \mathrm{~s}^{2} \mathrm{~S}_{1 / 2}-3 \mathrm{~d}{ }^{2} \mathrm{D}_{5 / 2}$ at 729 $\mathrm{nm}$ with natural linewidth of $0.2 \mathrm{~Hz}$ [16]. In our laboratory, a single ${ }^{40} \mathrm{Ca}^{+}$was trapped and laser-cooled in a miniature electric quadrupole Paul ring trap [17-21].

\subsection{Trap system}

(i) The miniature Paul trap. The trap has an endcap to center distance of $z_{0}=0.6 \mathrm{~mm}$ with a center to ring electrode distance $r_{0}=0.8 \mathrm{~mm}$. Two electrodes perpendicular to each

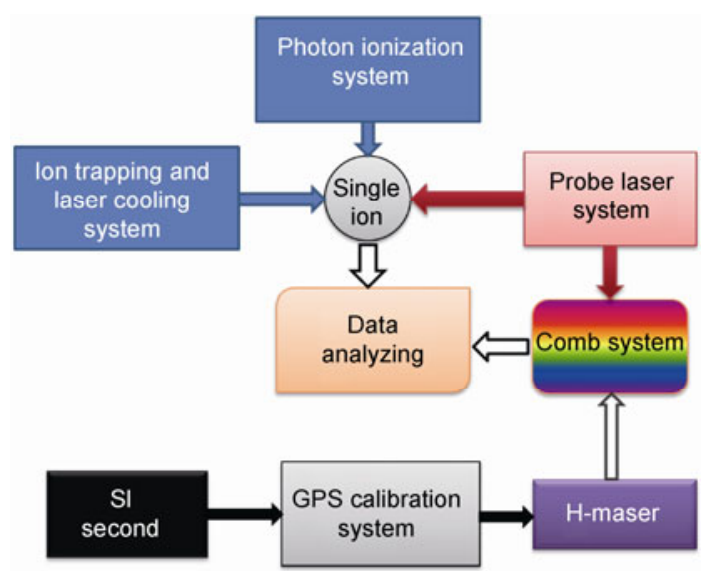

Figure 1 (Color online) Overall schematic diagram of the experimental setup.

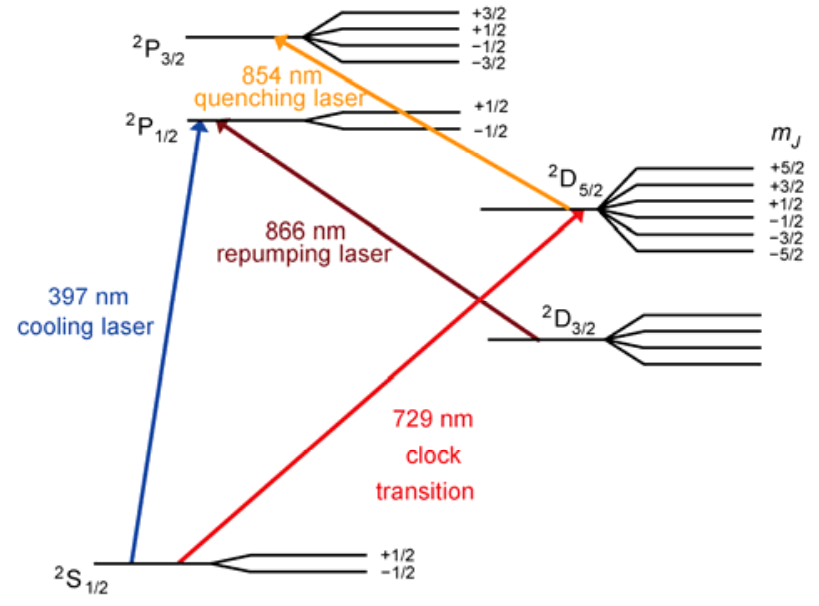

Figure 2 (Color online) Partial energy level diagram of ${ }^{40} \mathrm{Ca}^{+}$showing the principal transitions used in cooling, repumping and probing of the reference $729 \mathrm{~nm}$ transition.

other are set in the ring plane to compensate the ion's excess micromotion. The miniature structure makes for confining a single ion in the Lamb-Dick regime to eliminate the first-order Doppler shift; the nonstandard configuration compared to the classical Paul trap can reduce background noise. The trap is enclosed in a chamber that is evacuated to a pressure of less than $10^{-8} \mathrm{~Pa}$.

A trapping rf field with amplitude $350-650 \mathrm{~V}$ is applied to the ring at the frequency of $9.8 \mathrm{MHz}$. The excess micromotion is nulled by precisely adjusting the voltages on the endcap electrodes and the compensation electrodes.

(ii) Magnetic fields control. Variation of the ambient magnetic field strongly affects the precision of the optical frequency standard. When magnetic field is changing, the transition frequency also varies due to the linear Zeeman effect, and thus the transition linewidth is broadened to 10-100 times. Double layer magnetic shields are installed outside the ion trap vacuum chamber to shield the variation of the ambient magnetic field, and an attenuation of about 200 is achieved. A stable dc magnetic field of less than $1 \mu \mathrm{T}$ is applied after compensating the residual field by 3 pairs of coils perpendicular each other with 3 individual current source (YL4010\&YL4012, yltec). In such weak magnetic field, the ion cannot be effectively laser cooled with any specific polarization due to Hanle effect. An EOM (New focus) is used to modulate the polarization of the $866 \mathrm{~nm}$ repumping laser to avoid this effect.

(iii) Optical fields control. The clock transition is observed by the electron-shelving method [22]. A pulse-light sequence is introduced to observe the clock transition spectrum in order to avoid ac Stark shift by the 397, 866 and 854 $\mathrm{nm}$ radiations (Figure 3). The period of the $729 \mathrm{~nm}$ light is about $12 \mathrm{~ms}$, which induced a Fourier limit of a spectrum linewidth of about $100 \mathrm{~Hz}$. In the meantime, the 397, 866 and $854 \mathrm{~nm}$ lights are blocked. Then the state of the ion is interrogated using the cooling laser. If the count rate is 


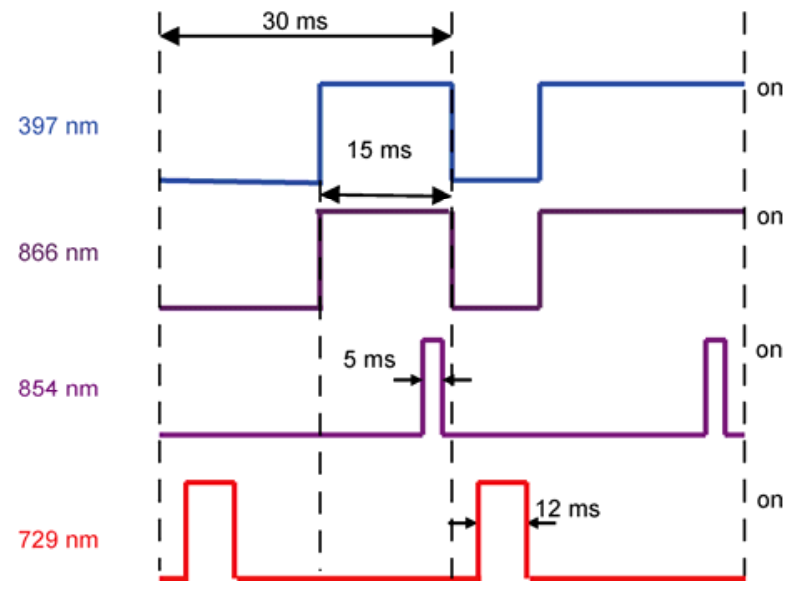

Figure 3 (Color online) Pulse-light sequence when observing the clock transition.

smaller than a fixed threshold, the clock transition takes place. After the interrogation, the ion is initialized again using the $854 \mathrm{~nm}$ laser.

\subsection{Laser systems}

(i) The lasers for ionization. Previously, the calcium ions were loaded by ionizing the neutral $\mathrm{Ca}$ atom beam with electron bombardment. This method is effective for loading ions. However, unexpected large heating rate is induced; the atoms deposited on the Paul trap electrodes near the ions would affect the ion storage. Recently photon ionization instead electron bombardment is used with a highly efficient loading scheme using a two-step photo-ionization scheme on a weak thermal beam of neutral atomic calcium [23]. The method avoids any charging of the nearby surroundings since no electron gun is used. Also, due to its extremely high efficiency, the atomic beam density can be greatly decreased, which allows one to reduce calcium deposited onto the trap electrodes from the oven. The schematic diagram for ion loading and laser cooling are shown in Figure 4. A home-made Littrow configuration diode laser is used to produce $846 \mathrm{~nm}$ laser with $\sim 100 \mathrm{~mW}$ of power, the laser beam is focused into a 5-mm-long PPKDP crystal and is frequency doubled with the second-harmonic generation (SHG), producing $423 \mathrm{~nm}$ laser with power of $\sim 10 \mu \mathrm{W}$. The $423 \mathrm{~nm}$ laser beam is focused into the ion trap together with a UV LED to carry out the photon ionization.

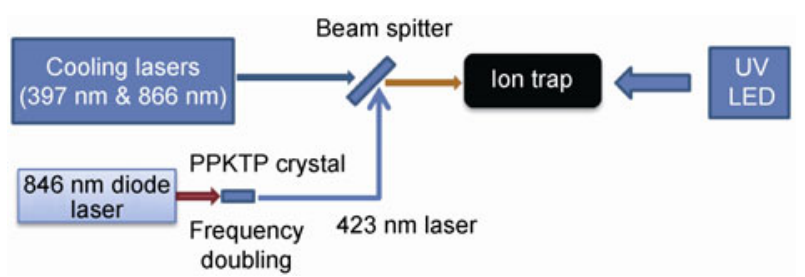

Figure 4 (Color online) The schematic diagram for ion loading and laser cooling. (ii) Laser frequency stabilization for cooling. To realize the optical frequency standard, effective trapping and cooling a single $\mathrm{Ca}^{+}$ion steadily in a Paul trap is an important precondition. Two lasers are needed in the process of laser cooling on $\mathrm{Ca}^{+}$. One is $397 \mathrm{~nm}$ cooling laser which pumps the ion from the $4^{2} \mathrm{~S}_{1 / 2}$ to the $3^{2} \mathrm{P}_{1 / 2}$ level. Another one is 866 $\mathrm{nm}$ radiation to drive the transition $3^{2} \mathrm{D}_{3 / 2}-4^{2} \mathrm{P}_{1 / 2}$ for avoiding the ion decays to $3^{2} \mathrm{D}_{3 / 2}$ metastable level.

The $397 \mathrm{~nm}$ laser diode from Nichia Corporation (NLHV3000E) is installed in the head of the commercial diode laser system DL100 (Toptica). The laser is a Littrow configuration. In the typical experimental condition ( $T=23.6^{\circ} \mathrm{C}, I=61 \mathrm{~mA}$ ), the output power of the laser is $\sim 30$ $\mathrm{mW}$. Its linewidth is $>4 \mathrm{MHz}$, and the long-term drift is $\sim 300 \mathrm{MHz}$ in $30 \mathrm{~min}$. In order to reduce the linewidth of the laser, we locked the laser to the transmission peak of a confocal Fabry-Perot interferometer with temperature stabilization (TOPTICA Photonics AG, FPI100). The free spectrum range (FSR) of FPI100 is $1 \mathrm{GHz}$, and its finesse is $\sim 400$. In this way, the linewidth of $397 \mathrm{~nm}$ laser is reduced to below $1 \mathrm{MHz}$ which is narrow enough for laser cooling of ${ }^{40} \mathrm{Ca}^{+}$ since the natural linewidth of $4^{2} \mathrm{~S}_{1 / 2}-3^{2} \mathrm{P}_{1 / 2}$ transition is $\sim 23$ $\mathrm{MHz}$.

When the $397 \mathrm{~nm}$ laser is only locked to FPI100, the laser still drifted several hundreds of $\mathrm{MHz}$ within half an hour with respect to the Optogalvanic (OG) signal. Therefore, we use the OG signal as a reference to control the cavity length of FPI100 using a computer program. Thus, the long-term drift of the laser is reduced to below $10 \mathrm{MHz}$ within $2 \mathrm{~h}$ [24]. The method for frequency stabilization of the laser at $866 \mathrm{~nm}$ is the same.

However, for the long-time-running of the optical frequency standard, both $397 \mathrm{~nm}$ and $866 \mathrm{~nm}$ lasers are stabilized to the $729 \mathrm{~nm}$ laser using transfer cavities [25]. The finesses of both cavities are more than 50 for the wavelengths of the diode laser and the probe laser $(729 \mathrm{~nm})$. The $729 \mathrm{~nm}$ laser is locked to a Zerodur cavity, which has a very low drift rate, and the diode lasers are coupled into two transfer cavities respectively, together with the $729 \mathrm{~nm}$ laser. The voltage added to the PZT is scanned with a frequency of $100 \mathrm{~Hz}$, and the transmission light of the cavity is detected by two photo diodes (PD). The signal from the PDs are then amplified and acquisitioned by computer using analog to digital convertor (ADC). By comparing the transmission fringes of the two lasers with different wavelength, we can calculate the relative drift rate of the diode laser referenced to the $729 \mathrm{~nm}$ laser, and then the error signal is fed back to the diode laser to stabilize the laser frequency. The frequency drift is reduced to less than $200 \mathrm{kHz}$ in $10 \mathrm{~h}$.

(iii) The ultra narrow linewidth probe laser. To observe the high- $Q$ optical clock transition with a natural linewidth of $0.2 \mathrm{~Hz}$, the probe laser's linewidth is expected to be $\mathrm{Hz}$ level, or even sub-Hz level. Two kinds of probe laser systems are developed, one is based on a commercial 
Ti:sapphire laser (MBR-110, Coherent), and another is based on a home-made diode laser with Littman configuration. The Ti:sapphire laser's wavelength can change in a large range continuously and is very stable when locked. In comparison the diode laser is much more compact in geometry and very convenient in use. They are both locked to a temperature-controlled high-finesse Fabry-Perot cavities using Pound-Drever-Hall (PDH) technique. The Ti:sapphire laser is locked to a Zerodur cavity placed on an active isolated platform (TS-140, Table stable) and the diode laser is locked to a ULE cavity with a football configuration placed on an passive isolated platform (BM-4, minus-K). A linewidth of about $13 \mathrm{~Hz}$ was measured from the heterodyne beatnote of the two lasers (Figure 5(a)). The long-term drift of the Ti:sapphire laser is $\sim 3 \mathrm{~Hz} / \mathrm{s}$ when locked using femto-second optical frequency comb referenced to a hydrogen maser (H-maser) (Figure 5(b)). An AOM (80 MHz, Brimrose) driven by a sweeping function generator (2023A, IFR) is used to compensate the long term drift; the compensation rate is changed every $300 \mathrm{~s}$ to compensate the nonlinear drift. After the compensation, we got a non-linear drift of below $0.1 \mathrm{~Hz} / \mathrm{s}$.

\subsection{The detection of the ion spectroscopy}

The PMT (EMI, 9893Q/100B) collects the weak fluorescence emitted by laser cooled ${ }^{40} \mathrm{Ca}^{+}$ion. The signal is amplified by a preamplifier (ORETEC, DC-200 MHz) and counted by a photon counter (Stanford Research System, SR400). The experiment processes are controlled by a personal computer program (LabVIEW 6.0). After ions are loaded in the trap, the $397 \mathrm{~nm}$ laser's frequency is slowly scanned across the resonance of $4^{2} \mathrm{~S}_{1 / 2}-4^{2} \mathrm{P}_{1 / 2}$ dipole transition, while the $866 \mathrm{~nm}$ laser stays at the resonance to prevent optical pumping to the $3^{2} \mathrm{D}_{3 / 2}$ level. Fluorescence profile of the ion cloud is obtained.

A single trapped ion is demonstrated and judged by the electron shelving technique. Quantum jumps profile as a substitution is adopted to investigate the quadrupole transition, which is obtained by scanning the $729 \mathrm{~nm}$ laser's frequency and recording the numbers of quantum jumps. The closer of the $729 \mathrm{~nm}$ laser's frequency to each component's resonance point, the more quantum jumps are detected at the same probing time or the same numbers of probe laser pulses.

\subsection{The optical frequency comb as a link with optical and microwave frequencies}

A commercial Ti:sapphire based optical frequency comb (FC 8004, MenloSystems) is used to measure the $729 \mathrm{~nm}$ laser frequency. A mode-locked Ti:sapphire laser pumped by $6 \mathrm{~W}$ laser at $532 \mathrm{~nm}$ (Verdi V-6, Coherent) produce fs pulses (normally $30 \mathrm{fs}$ ) at a repetition rate of approximately $200 \mathrm{MHz}$. The frequency of the $n$-th comb component can be expressed as $f_{n}=n \times f_{\text {rep }}+f_{\text {CEO }}[26,27]$, where $f_{\text {rep }}$ is the repetition rate of the laser pulses and $f_{\mathrm{CEO}}$ is the carrier-envelope offset frequency. The output is focused into two PCFs (photon crystal fiber), one for the observation of the offset frequency detection and another for the probe laser measurement. The spectrum is normally broadened to over an octave after the fiber, from approximately $500 \mathrm{~nm}$ to $1100 \mathrm{~nm}$. A self-referencing system with an f-to-2f interferometer is introduced for the offset frequency detection. The infrared part of the broadened comb beam is separated with a dichroic mirror and frequency doubled with the SHG using a 5-mm-long $\mathrm{KNbO}_{3}$ crystal, and then overlapped with the green part of the broadened beam using a polarized beam splitter (PBS). The signal to noise ratio $(\mathrm{S} / \mathrm{N})$ of the carrier-envelope beat frequency is $40 \mathrm{~dB}$ at a resolution bandwidth of $300 \mathrm{kHz}$. Both the repetition frequency and the offset frequency are locked to two individual synthesizers, which are referenced to a $10 \mathrm{MHz}$ signal provided by an active $\mathrm{H}$-maser (CH1-75A) with an isolated splitter and a
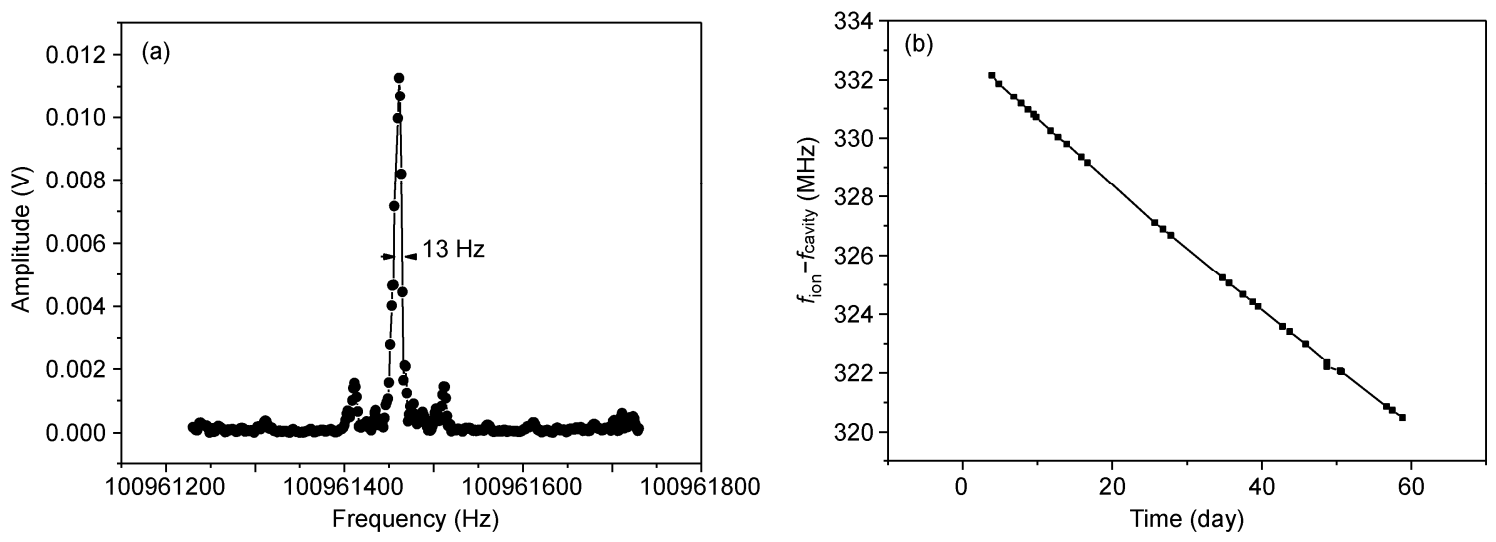

Figure 5 (a) A $13 \mathrm{~Hz}$ linewidth beatnote of the Ti:sapphire laser locked to the Zerodur cavity and the diode laser locked to the ULE cavity was measured using a photon detector and a spectrum analyzer (Agilent E4405B) with a resolution bandwidth of $3 \mathrm{~Hz}$; (b) cavity frequency drift measured with the $\mathrm{H}$-maser and the comb with several tens of days of measurements shows a long term linear drift of about $3 \mathrm{~Hz} / \mathrm{s}$. 
60-m-long standard $50 \mathrm{Ohm}$ coaxial cable (RG-213). The output of the comb laser from the other PCF (with frequency of $f_{n}$ ) is overlapped with the probe laser at $729 \mathrm{~nm}$ (with frequency of $f_{\mathrm{c}}$ ) using a PBS. The beat frequency $f_{\mathrm{b}}=\left|f_{\mathrm{c}}-f_{n}\right|$ between the probe laser and the $n$-th comb component at $729 \mathrm{~nm}$ is measured by two individual frequency counters referenced to the H-maser. Normally the $\mathrm{S} / \mathrm{N}$ of the beat frequency is $30 \mathrm{~dB}$ at a resolution bandwidth of $300 \mathrm{kHz}$. However, sometimes the $\mathrm{S} / \mathrm{N}$ of the frequency dropped to less than $28 \mathrm{~dB}$ during the measurement so that the readings are not reliable. We use two individual counters measuring the beat frequency simultaneously, if the difference of the readings of the two counters are more than $1 \mathrm{~Hz}$, we believe the measurement is not reliable and the measurement is not taken into count. The probe laser frequency is measured every $1 \mathrm{~s}$.

\section{Experiments}

\subsection{The trapping and cooling of a single ion}

Full details of the laser cooling, trapping, detecting and probing system used in this work are reported in previous work $[17,18]$.

First, the trapping rf field is applied to the ring electrode, and then the ions are loaded using either electron bombardment or photon ionization. Then, by scanning cooling laser from red detuning to resonance center, we can get the fluorescence spectrum. Typically, approximately $10 \mu \mathrm{W}$ of the $397 \mathrm{~nm}$ laser power is focused on the single ion with a spot size of about $40 \mu \mathrm{m}, 600 \mu \mathrm{W}$ of power with $60 \mu \mathrm{m}$ of size for the $866 \mathrm{~nm}$ laser. Normally, more than one ion is trapped when just loaded. The cooling lasers are blocked for several times to get a single ion.

The secular motion is observed by two ways in ion cloud and a single ion system separately. One is the method of RF field resonance. An additional RF voltage (Stanford Re- search System, DS345) of $2 \mathrm{~V}$ (peak to peak) is added to one of the endcaps and compensation electrodes respectively, and then we manually scan the frequency of the additional RF to look for a drop in the fluorescence level; the other one is observing secular motion sidebands of a single ${ }^{40} \mathrm{Ca}^{+}$ion's $4^{2} \mathrm{~S}_{1 / 2}-3^{2} \mathrm{D}_{5 / 2}$ transition Zeeman profile. Based on the measurements, the secular frequencies of the trap are $\omega_{x}$ $\approx \omega_{y} \approx 700 \mathrm{kHz}$ and $\omega_{z} \approx 1.5 \mathrm{MHz}$, respectively.

The single ion suffers the excess micromotion. The amplitude of ion micromotion strongly depends on additional static electric field. Two methods of detecting ion micromotion are demonstrated. One relies on the alterations of atomic transition line shape; another depends on the rfphoton correlation technique [28]. The micromotion is minimized before each measurement with the observation by the rf-photon correlation method by adjusting the endcap and compensation electrode voltages.

The single ion fluorescence line shape and quantum jump signal are optimized by patient and repeated experiments step by step recent years. The new results are much better than ever before; a typical counting rate of $7000 \mathrm{~s}^{-1}$ is observed for the single cold ${ }^{40} \mathrm{Ca}^{+}$(Figure 6). The ion can stay in the trap without any laser for up to $50 \mathrm{~h}$.

\subsection{Observation of the clock transition and lock the probe laser to the transitions}

The frequency difference between the probe laser and the clock transition line center of the ion is cancelled by an AOM. The required AOM frequencies are updated every 40 cycles of pulses, which cost about $1.5 \mathrm{~s}$. By the "4 points locking scheme" [28,29], 3 pairs of the Zeeman transitions $\left(M_{J}= \pm 1 / 2, M_{J}= \pm 3 / 2\right.$ and $\left.M_{J}= \pm 5 / 2\right)$ are interrogated to cancel the electric quadrupole shift [30,31], and the offset frequency $\Delta f(i)$ between the probe laser and the transition could be obtained every $13 \mathrm{~s}$ (Figure 7(a)). However, it is not easy to lock the probe laser to three pairs of the Zeeman
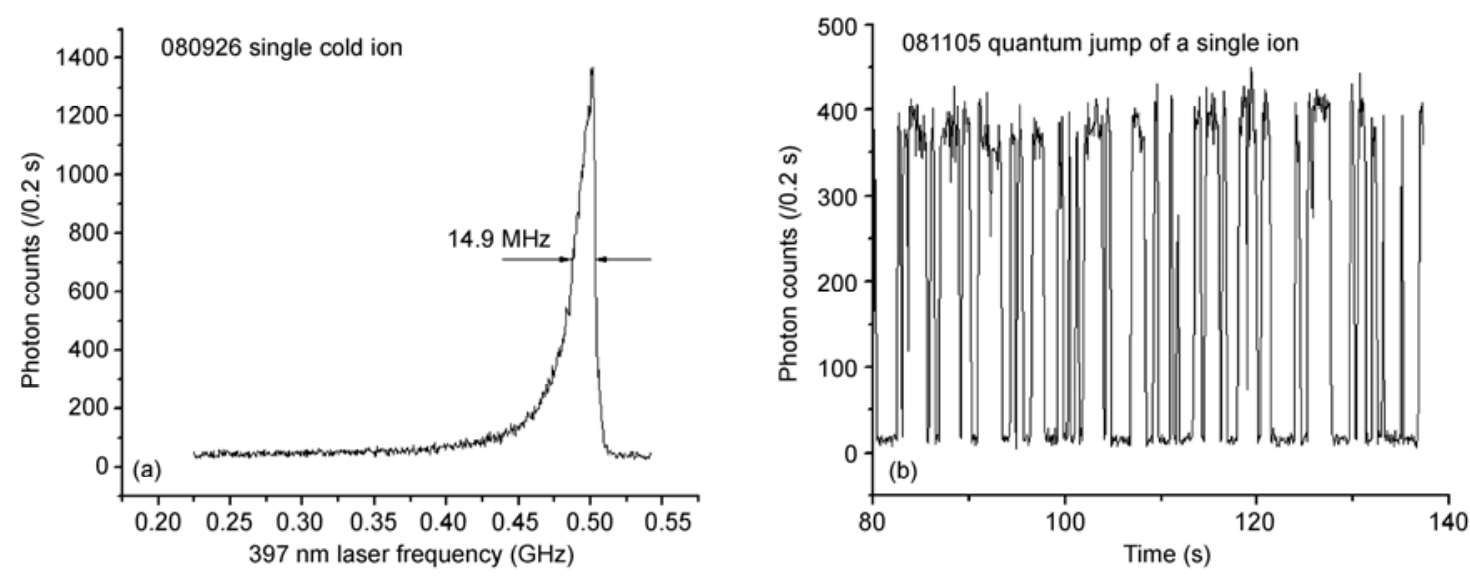

Figure 6 (a) The line width of single ion fluorescence signal is about $14.9 \mathrm{MHz}$ and photon counts rate is up to $7 \mathrm{kHz}$; (b) single ion quantum jump signal with a signal-to-noise better than 50 in $200 \mathrm{~ms}$. 

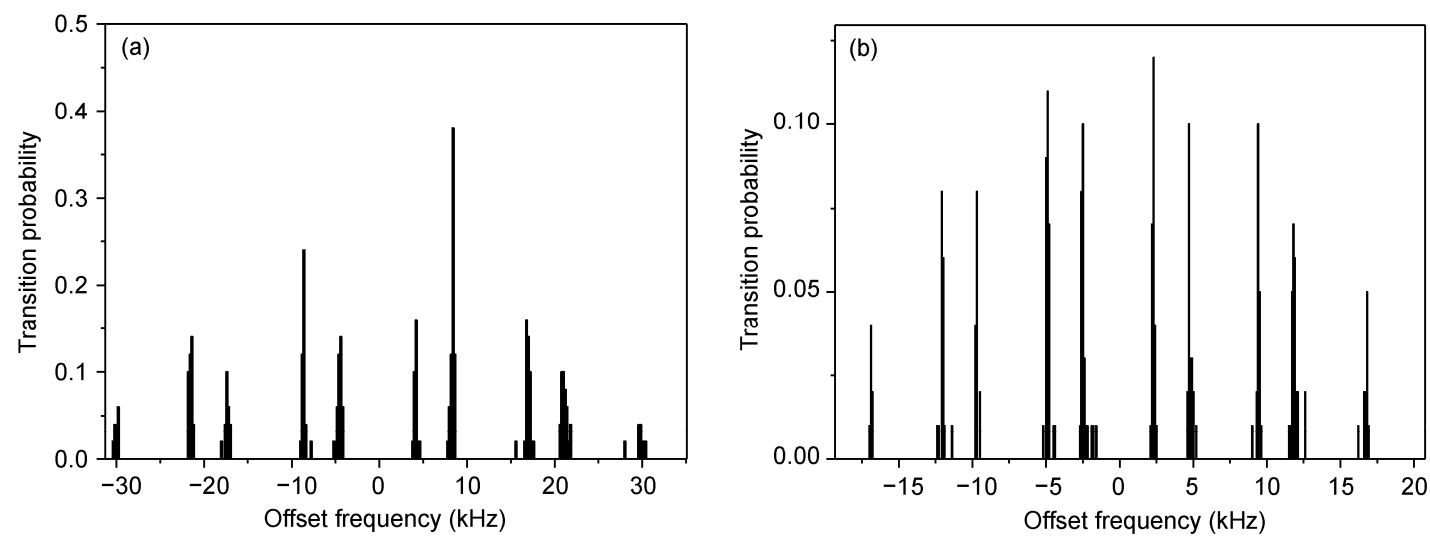

Figure 7 (a) Ten components of the Zeeman profile before adjusted; (b) ten components of the Zeeman profile after adjusted.

components synchronously, since the relative intensities of the observed Zeeman components are usually different (Figure 7(a)) and sometimes the probability of some of the components are very small, and thus the locking of the laser to the ion transitions is unstable. In order to get a perfect lock, the probability of the Zeeman components which we choose are required to be equal. In practice, the magnetic field is firstly compensated to be smaller than $10 \mathrm{nT}$. Then the proper angle between the direction of propagation of the laser and the direction of $\boldsymbol{B}$ field is achieved by changing the current of the three pairs of coils. Finally the polarization of the $729 \mathrm{~nm}$ laser beam is adjusted to get a best profile of the transitions (Figure 7(b)).

Typically, approximately $150 \mu \mathrm{W}$ of the $854 \mathrm{~nm}$ laser power is focused on the single ion with a spot size of about $200 \mu \mathrm{m}$, with a typical linewidth of $10 \mathrm{MHz}$; and $40 \mathrm{nW}$ of the $729 \mathrm{~nm}$ laser power is focused with waist size of about $200 \mu \mathrm{m}$.

\section{Evaluation of the systematic shifts for the ${ }^{40} \mathrm{Ca}^{+}$clock transition}

There are a variety of potential sources of systematic shifts for the quadrupole $729 \mathrm{~nm} 4 \mathrm{~s}^{2} \mathrm{~S}_{1 / 2}-3 \mathrm{~d}^{2} \mathrm{D}_{5 / 2}$ clock transition in a trapped and laser cooled ${ }^{40} \mathrm{Ca}^{+}$. We shall consider the systematic effects: second order Doppler effect, quadratic Stark shift (DC and AC), electric quadrupole shift, blackbody Stark shift, quadratic Zeeman shift, and gravitational potential. The detailed study of systematic shifts on ${ }^{40} \mathrm{Ca}^{+}$ clock transition was reported in $[32,33]$.

\subsection{Second order Doppler shift}

The second order Doppler shift is caused by the relativistic Doppler effect, due to the ion motion relative to the laboratory frame, with bothering by the thermal kinetic energy and the micromotion. First of all, the micromotion should be minimized for achieving lower temperature and could be measured by rf-photon correlation method. After the mini- mization of the micromotion, we can do the measurements. For our ring trap, we measure the ion temperature by observing the intensity of secular sidebands. We obtain ion temperature of 3(3) $\mathrm{mK}$ by calculation [32]. With the temperature estimated, the second Doppler shift caused by thermal contribution is estimated to be $-0.004(0.004) \mathrm{Hz}$ [34]. For the second Doppler shift caused by micromotion, it can be estimated by observing the intensity of the micromotion sidebands relative to the carrier or observing the cross-correlation signal [34]. From the correlation signal observed, typically with a modulation amplitude ratio of $0.2(0.1)$, considering that the direction of the micromotion is not well known, the shift is estimated to be $-0.02(0.02)$ $\mathrm{Hz}$.

\subsection{Stark shift due to micromotion, thermal motion and blackbody radiation}

Thermal secular motion and excess micromotion can push the ion out of saddle point of the trap, which introduce a Stark shift. The Stark shift due to micromotion can be calculated from the ion temperature above that for the three pair of transitions [34], and our total averaged Stark shift due to micromotion is less than $1 \mathrm{mHz}$. The Stark shift due to thermal motion, for the three pair of components as above, we can calculate that the total averaged shift is also less than $1 \mathrm{mHz}$ from the correlation signal [34]. There is also a Stark shift arising from blackbody radiation. At a room temperature of $293 \mathrm{~K}$, assuming the real temperature fluctuation is $2 \mathrm{~K}$, the shift is $0.35(0.02) \mathrm{Hz}$ [35].

\subsection{Ac Stark shift due to the cooling laser, the repum- ng laser, the quenching laser and the probe laser}

In our experiment, during the interrogation time, all the laser beams are switched off by mechanical shutter and AOM except for the $729 \mathrm{~nm}$ laser. We have measured the real efficiency of the shutter and the AOM, the shutter is better than $70 \mathrm{~dB}$ of the attenuation and for the AOM is better than $40 \mathrm{~dB}$. 
The radiations used to cool and probe the trapped ion can cause ac Stark shifts of the clock transition frequency. For the $397 \mathrm{~nm}$ laser, a shutter and an AOM are used to switch off the laser beam. The frequency difference between with AOM always on and with AOM off when doing the interrogations with the $729 \mathrm{~nm}$ laser is measured to be less than $10 \mathrm{~Hz}$. Therefore, with an attenuation of better than $40 \mathrm{~dB}$ for the AOM which switches off the $397 \mathrm{~nm}$ radiation when the measurements are made, the shift is less than $1 \mathrm{mHz}$. For the laser at $866 \mathrm{~nm}$, a shutter is used to switch off the light; the frequency difference of less than $30 \mathrm{~Hz}$ between with shutter always on and with shutter off when doing the interrogations with the $729 \mathrm{~nm}$ laser is measured. Therefore, with an attenuation of better than $70 \mathrm{~dB}$ for the shutter which switches off the $866 \mathrm{~nm}$ radiation when the measurements are made, the shift is less than $1 \mathrm{mHz}$. For the 854 $\mathrm{nm}$ laser beam, two individual shutters are used to block the light. A frequency difference between with $854 \mathrm{~nm}$ laser off and with only one shutter off when doing the interrogations with $729 \mathrm{~nm}$ laser is measured to be less than $20 \mathrm{~Hz}$. Therefore, with an attenuation of better than $70 \mathrm{~dB}$ for the other shutter which switches off the $854 \mathrm{~nm}$ radiation when the measurements are made, the shift should be less than 1 $\mathrm{mHz}$. The ac Stark shift caused by the $729 \mathrm{~nm}$ laser is measured by doing measurements at different probe laser intensities. From the experiment results, we obtain a linear fit slope of $0.04(0.06) \mathrm{Hz} / I$, where $I$ is the typical intensity used for the measurements.

\subsection{Zeeman Shift}

The linear Zeeman effect can be effectively eliminated by locking to a pair of Zeeman components which are symmetrically placed around line center. However, there may be ac broadening of the components and the fast changes of the dc magnetic field could cause a locking problem. As for the long term dc magnetic field drift as well as the probe laser cavity drift, we can estimate the shifts using the servo error signal. In our case, the servo error would be $0.11(0.03) \mathrm{Hz}$. As for the second order Zeeman shift, it can be calculated using second order perturbation theory. For our system, the average magnetic field during the measurements is $430 \mathrm{nT}$ and the fluctuation of the field we measured is about $3 \mathrm{nT}$. This leads to a second Zeeman shift of less than $1 \mathrm{mHz}$, which is negligible.

\subsection{Electric quadruple Shift}

There will be an electric quadrupole shift due to the presence of electric field gradients, which interact with the electric quadrupole moment of the ion. However, by averaging the center frequency of the three pairs of the components, we can null the quadrupole shift [32]. According to the magnetic field drift rate we measured, normally less than 1 nT per hour, the 6 seconds of measuring time difference can induce a shift error of less than $0.02 \mathrm{~Hz}$. By averaging the difference of center frequency for different components, an error of $0.03 \mathrm{~Hz}$ is obtained.

\subsection{Gravitational Shift}

There will be a gravitational shift due to the gravitation. Different altitude would introduce different shift. We measured the altitude of our ion trap referenced to the sea level using GPS. The measured results was 35.2(1.0) $\mathrm{m}$, and thus the gravitational shift for the clock is estimated to be 1.583(0.045) Hz.

Table 1 shows the total summary of the frequency shifts considered. Systematic shifts with less than $10^{-18}$ of the affection are not included. Taking into account all of them, we get a total fractional shift of $4.74 \times 10^{-15}$ with a fractional uncertainty of $5.0 \times 10^{-16}$ from the data obtained in May 2011 and a total fractional shift of $4.74 \times 10^{-15}$ with a fractional uncertainty of $6.5 \times 10^{-16}$ for the data obtained in June 2011 .

Table 1 The systematic frequency shifts and their uncertainties of the evaluation of the clock. Shifts and uncertainties given are in fractional frequency units $(\Delta v / v)$; shifts with less than $10^{-18}$ of the affection are not included

\begin{tabular}{|c|c|c|c|c|}
\hline \multirow{2}{*}{ Effect } & \multicolumn{2}{|c|}{ Measurements in May } & \multicolumn{2}{|c|}{ Measurements in June } \\
\hline & Shift $\left(10^{-16}\right)$ & Uncertainty $\left(10^{-16}\right)$ & Shift $\left(10^{-16}\right)$ & Uncertainty $\left(10^{-16}\right)$ \\
\hline 2nd order Doppler shift due to thermal motion & -0.10 & 0.10 & -0.10 & 0.10 \\
\hline 2nd order Doppler shift due to micromotion & -0.49 & 0.49 & -0.49 & 0.49 \\
\hline Stark shift due to thermal motion and micromotion & 0 & 0.04 & 0 & 0.04 \\
\hline ac Stark shift due to $397 \mathrm{~nm}, 866 \mathrm{~nm}$ and $854 \mathrm{~nm}$ & 0 & 0.04 & 0 & 0.04 \\
\hline ac Stark shift due to $729 \mathrm{~nm}$ & 0.97 & 1.46 & 0.97 & 1.46 \\
\hline Blackbody radiation shift & 8.51 & 0.27 & 8.51 & 0.27 \\
\hline Linear Zeeman shift & 0 & 4.52 & 0 & 6.23 \\
\hline Electric quadrupole shift & 0 & 0.72 & 0 & 0.51 \\
\hline Gravitational shift & 38.44 & 1.10 & 38.44 & 1.10 \\
\hline Total shift & 47.4 & 5.0 & 47.4 & 6.5 \\
\hline
\end{tabular}


4 Absolute frequency measurement of the ${ }^{40} \mathrm{Ca}^{+}$ clock transition with respect to the SI second through GPS

\subsection{Frequency measurement based on a H-maser}

The probe laser frequency measured with the comb $f_{\mathrm{c}}(i)$ could be calculated using the formula $f_{\mathrm{c}}=n \times f_{\mathrm{rep}} \pm f_{\mathrm{CEO}} \pm f_{\mathrm{b}}$. The integer number $n$ could be calculated using a wavemeter with an accuracy of less than $100 \mathrm{MHz}$, and the ambiguous signs could be removed by observing the sign of the variation in the beat frequency in the beat frequency while the repetition frequency or the carrier-envelope offset frequency was changed. Figure 8(b) shows the probe laser frequency $f_{\mathrm{c}}(i)$ measured with the comb referenced to the $\mathrm{H}$-maser. The observed short-term frequency noise is mainly contributed by the H-maser through the 60-m-long cable and the $20 \mathrm{MHz}$ synthesizer that was used in locking the $f_{\text {rep }}$ to the H-maser.

Using the two sets of the AOM offset frequency (Figure $8(\mathrm{a})$ ) and the measured probe laser frequency (Figure 8(b)), we calculated the clock transition frequency to be $v_{0}(i)=$ $f_{\mathrm{c}}(i)+\Delta f(i)$ for $i=1,2, \ldots i_{\max }$, where $i_{\max }$ represents the total measurements number. In the case shown in Figure 8, the total measurement number is about 3500. Figure 8(c) shows the calculated frequency data sets of $v_{0}(i)$, which gives an
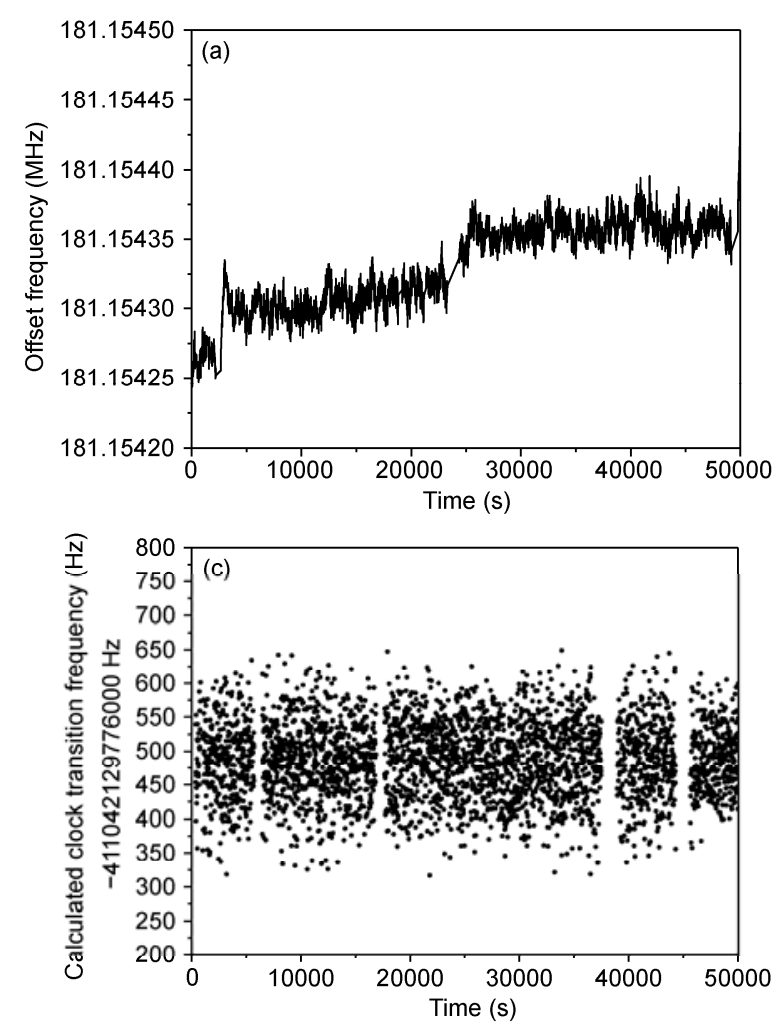

averaged value $v_{0}=411042129776490.7 \mathrm{~Hz}$. The histogram of the $v_{0}(i)$ (Figure $8(\mathrm{~d})$ ) follows a normal distribution; the standard deviation of the mean $\sigma / \sqrt{i_{\max }}$ is $3.5 \mathrm{~Hz}$.

Frequency measurements were taken in 32 individual days, separated into two parts, one in May 2011 with 15 continuous days and the other in June 2011 with 17 continuous days (Figure 9). Each filled circles in Figure 9 represents a mean value of $v_{0}(i)$ whose measurement was based directly on the H-maser. The error bars are given by the standard deviation of the mean $\sigma / \sqrt{i_{\max }}$. The former 15 days of measurements gives a weighted averaged frequency of $411042129776489.7(0.9) \mathrm{Hz}$ and the later 17 days of measurements gives a weighted averaged frequency of 411042129776489.1(0.4) Hz. The measurement described in Figure 8 corresponds to the measurement in MJD day 55726.

\subsection{Determination of the absolute frequency of the $4 \mathrm{~s}$ ${ }^{2} S_{1 / 2}-3 d{ }^{2} D_{5 / 2}$ clock transition}

To get the final absolute frequency measurement of the clock transition, systematic shifts and the calibration of the reference must be considered and applied to the above averaged frequency. To calibrate the frequency of the H-maser, a GPS receiver with an antenna (TTS-4, PikTime Systems)
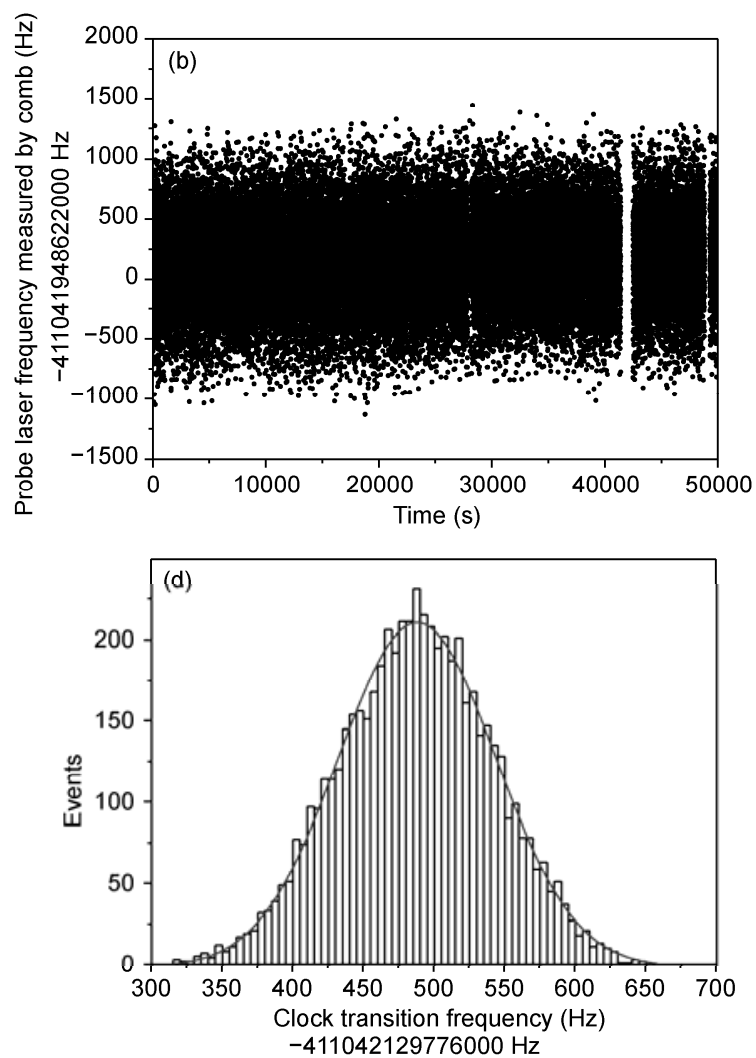

Figure 8 (a) Measured AOM offset frequency $\Delta f(i)$; (b) measured probe laser frequency $f_{\mathrm{c}}(i)$ using the comb referenced to the H-maser; (c) frequency of the clock transition $v_{0}(i)$ calculated from $\Delta f(i)$ and $f_{\mathrm{c}}(i)$; (d) histogram of the $v_{0}(i)$ with a Gaussian fitting. 


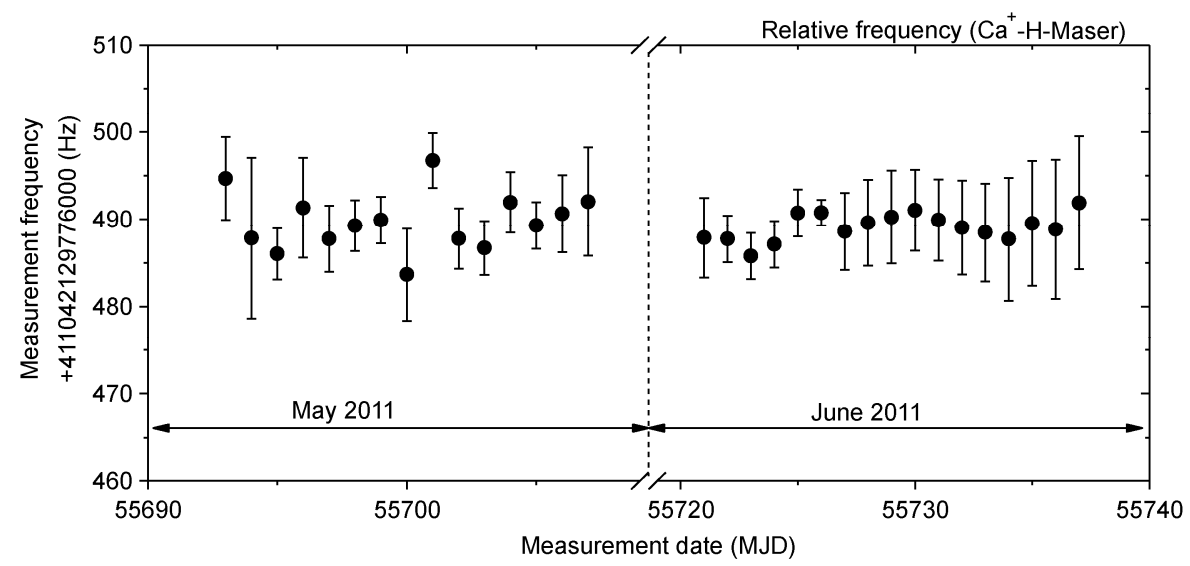

Figure 9 Frequency measurement of the $4 \mathrm{~s}^{2} \mathrm{~S}_{1 / 2-}-3 \mathrm{~d}^{2} \mathrm{D}_{5 / 2}$ transition of a laser cooled trapped single ${ }^{40} \mathrm{Ca}^{+}$reference to the H-maser. Data shown in this figure do not include systematic corrections.

is used. A detailed report on how the H-maser was calibrated using GPS can be found in [34]. Briefly, with cooperation with National Institute of Metrology of China (NIM), the H-maser frequency were calibrated using precise point positioning (PPP) technique, with help of GPS satellites and the frequency comparison data published in BIPM website [36] every month. Table 2 shows the estimation for corrections to the absolute frequency of the ${ }^{40} \mathrm{Ca}^{+}$clock transition.

Based upon the data listed in Table 2, we determine that the total correction for the frequency measurement shown in Figure 8 is $-96.9 \mathrm{~Hz}$ for the data obtained in May 2011 and $-96.4 \mathrm{~Hz}$ for the data obtained in June 2011. The combined fractional uncertainty of the absolute frequency measurement is $4.6 \times 10^{-15}$ for the data obtained in May 2011 and $2.6 \times 10^{-15}$ for the data obtained in June 2011. The corrected absolute frequency of the ${ }^{40} \mathrm{Ca}^{+} 4 \mathrm{~s}^{2} \mathrm{~S}_{1 / 2}-3 \mathrm{~d}{ }^{2} \mathrm{D}_{5 / 2}$ clock transition is $411042129776393.3(1.9) \mathrm{Hz}$ for the data obtained in May 2011 and 411042129776392.7(1.1) Hz for the data obtained in June 2011. The two measurements agree with each other within their uncertainties. The unweighted mean of the above two values gives a final result of 411042129776393.0(1.6) Hz, and the final uncertainty is calculated by considering both statistical and systematical uncertainties. The obtained final result of absolute frequency measurement of the ${ }^{40} \mathrm{Ca}^{+} 4 \mathrm{~s}^{2} \mathrm{~S}_{1 / 2}-3 \mathrm{~d}{ }^{2} \mathrm{D}_{5 / 2}$ clock transition is $411042129776393.0(1.6) \mathrm{Hz}$, which agree with the former measurements $[9,14,37]$ by both University of Inns- bruck and the NICT (Figure 10).

\subsection{Discussion of the methods measuring the transition}

We measure the absolute frequency of the clock transition twice: one in May 2011 and the other in June 2011; thus we obtain two measurement results: one is 411042129776393.3 (1.9) $\mathrm{Hz}$ and the other is $411042129776392.7(1.1) \mathrm{Hz}$. The weighted mean of the above two would be 411042129776392.9(1.0) $\mathrm{Hz}$ and the unweighted mean would be $411042129776393.0(1.6) \mathrm{Hz}$. The difference of two results is much smaller than the error, and thus using any result would be fine.

\section{Conclusion}

We have performed systematic frequency measurements of the ${ }^{40} \mathrm{Ca}^{+}$clock transition at $10^{-15}$ level with a laser cooled single ion in a miniature Paul trap. We use GPS to calibrate our H-maser, and to approach a measurement accuracy level of $10^{-15}$ and we perform the measurement for a long averaging time (32 days with more than 2 million seconds of the measurements). With the GPS PPP technique, we achieve a frequency transfer uncertainty of about $1 \times 10^{-14}$ with an averaging time of one day. The total systematic error for the clock transition frequency is better than $6.5 \times 10^{-16}$. To

Table 2 The absolute frequency measurement budget table. The unit of shifts and uncertainties are given in $\mathrm{Hz}$

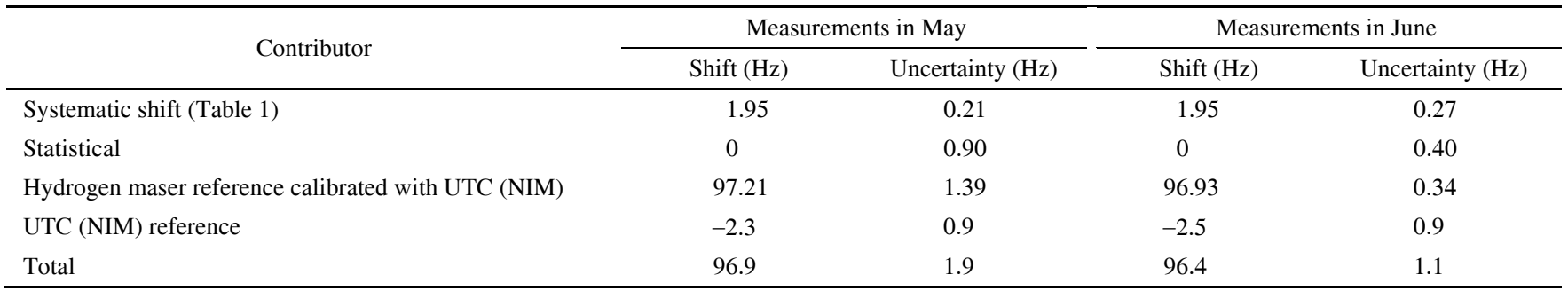




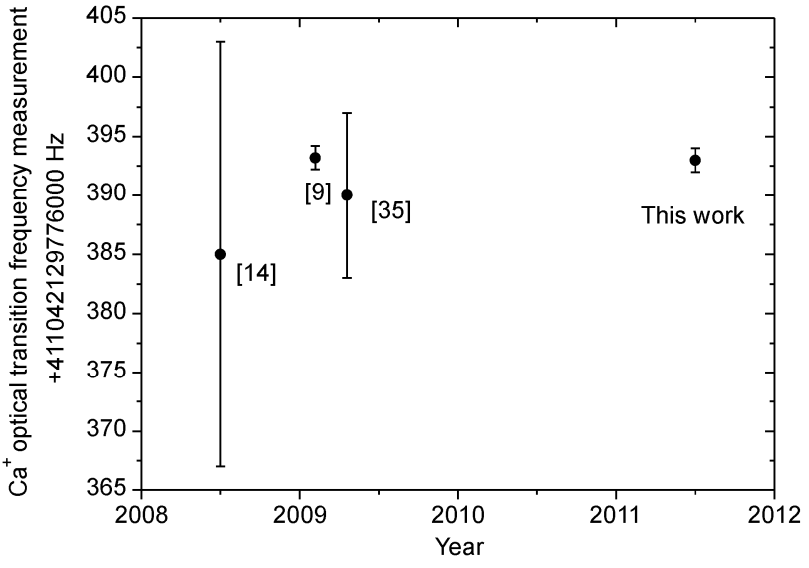

Figure 10 Comparison of the ${ }^{40} \mathrm{Ca}^{+}$clock transition frequency measured by University of Innsbruck [9], the NICT [14,37] and the results in this paper.

achieve a smaller uncertainty, we will need to use a more stable reference to reduce the statistic error. We find that the uncertainty of linear Zeeman shift is in fact limiting the final systematic uncertainty. The linear Zeeman effect uncertainty is mainly caused by the fluctuation of the magnetic field, which is measured by calculating the variance of the Zeeman splitting of the Zeeman transitions. According to the variance of the Zeeman splitting, the uncertainty is calculated from statistics.

To reduce the systematic uncertainties in the future, one has to increase the stability of the magnetic field. Next step in the near future, we will set up the second similar ion trap to do the comparison between two frequency standards. With the comparison, we expect the Allan deviation can reduce to $10^{-16}$ level. We also would like to do the comparison between us and NICT or University of Innsbruck by GPS. A Cs fountain may be introduced to do a new round of measurement to make the result even better, approaching $10^{-16}$ level.

It is very grateful for $Y$. Huang and H. Guan's organizing and amending this paper. We acknowledge Y. Huang, J. Cao, P. Liu, H. Guan, X. Huang, Q. Liu, B. Guo, B. Ou, G. Huang (NPL), H. Shu, H. Fan, J. Li and X. Zhu for processing the works, and Z. Wei (IOP), L. Kun (NIM), T. Li (NIM) for cooperation work. J. Ye (JILA), Y. Li (NICT), K. Matsubara (NICT), H. Klein (NPL), L. Ma (ECNU), Y. Wang (Peking University), Y. Wang (SIOM), C. Ye (WIPM), M. Zhan (WIPM), Z. Yan (UNB) and C. Lee (SYSU) for their fruitful discussion and the valuable suggestion. This work was supported by the National Basic Research Program of China (2005CB724502 and 2012CB821301), National Natural Science Foundation of China (10274093, 60490282, 10874205 and 11034009) and Chinese Academy of Sciences.

1 Li R X, Gibble K, Szymaniec K. Improved accuracy of the NPLCsF2 primary frequency standard: Evaluation of distributed cavity phase and microwave lensing frequency shifts. Metrologia, 2011, 48: 283-289

2 Campbell G K, Ludlow A D, Blatt S, et al. The absolute frequency of the ${ }^{87}$ Sr optical clock transition. Metrologia, 2008, 45: 539-548
3 Akatsuka T, Takamoto M, Katori H. Optical lattice clocks with non-interacting bosons and fermions. Nat Phys, 2008, 4: 954-959

4 Lemke N D, Ludlow A D, Barber Z W, et al. Spin-1/2 optical lattice clock. Phys Rev Lett, 2008, 103: 063001

5 Poli N, Barber Z W, Lemke N D, et al. Frequency evaluation of the doubly forbidden ${ }^{1} \mathrm{~S}_{0} \rightarrow{ }^{3} \mathrm{P}_{0}$ transition in bosonic ${ }^{174} \mathrm{Yb}$. Phys Rev A, 2008, 77: 050501

6 Margolis H S, Barwood G P, Huang G, et al. Hertz-level measurement of the optical clock frequency in a single ${ }^{88} \mathrm{Sr}^{+}$ion. Science, 2004, 306: 1355-1358

7 Huntemann N, Okhapkin M, Lipphardt B, et al. High-accuracy optical clock based on the octupole transition in ${ }^{171} \mathrm{Yb}^{+}$. Phys Rev Lett, 2012, 108: 090801

8 Rosenband T, Hume D B, Schmidt P O, et al. Frequency ratio of $\mathrm{Al}^{+}$ and $\mathrm{Hg}^{+}$single-ion optical clocks; Metrology at the 17th Decimal Place. Science, 2008, 319: 1808-1812

9 Chwalla M, Benhelm J, Kim K, et al. Absolute frequency measurement of the ${ }^{40} \mathrm{Ca}^{+} 4 \mathrm{~s}^{2} \mathrm{~S}_{1 / 2}-3 \mathrm{~d}{ }^{2} \mathrm{D}_{5 / 2}$ clock transition. Phys Rev Lett, 2009, 102: 023002

10 Stalnaker J E, Diddams S A, Fortier T M, et al. Optical-to-microwave frequency comparison with fractional uncertainty of $10^{-15}$. Appl Phys B, 2007, 89: 167-176

11 Chou C W, Hume D B, Koelemeij J C J, et al. Frequency comparison of two high-accuracy $\mathrm{Al}^{+}$optical clocks. Phys Rev Lett, 2010, 104: 070802

12 The International Committee for Weights and Measures (CIPM). Recommendation 2 (c2-2009)-(CIPM): Updates to the list of standard frequencies

13 Chen J B. Active optical clock. Chin Sci Bull, 2009, 54: 348-352

14 Matsubara K, Hayasaka K, Li Y, et al. Frequency measurement of the optical clock transition of ${ }^{40} \mathrm{Ca}^{+}$ions with an uncertainty of $10^{-14}$ level. Appl. Phys Express, 2008, 1: 067011

15 Champenois C, Houssin M, Lisowski C, et al. Evaluation of the ultimate performances of a $\mathrm{Ca}^{+}$single-ion frequency standard. Phys Lett A, 2004, 331: 298-311

16 Barton P A, Donald C J S, Lucas D M, et al. Measurement of the lifetime of the $3 d^{2} D_{5 / 2}$ state in ${ }^{40} \mathrm{Ca}^{+}$. Phys Rev A, 2000, 62: 032503

17 Shu H L, Guan H, Huang X R, et al. A single laser cooled trapped ${ }^{40} \mathrm{Ca}^{+}$ion in a miniature Paul trap. Chin Phys Lett, 2005, 22: 16411644

18 Shu H L, Guo B, Guan H, et al. Experimental improvement of signal of a single laser-cooled trapped ${ }^{40} \mathrm{Ca}^{+}$ion. Chin Phys Lett, 2007, 24 : 1217-1219

19 Guo B, Guan H, Liu Q, et al. Preliminary frequency measurement of the electric quadrupole transition in a single laser-cooled ${ }^{40} \mathrm{Ca}^{+}$ion. Front Phys China, 2009, 4: 144-154

20 Liu Q, Huang Y, Cao J, et al. Frequency measurement of the electric quadrupole transition in a single laser-cooled ${ }^{40} \mathrm{Ca}^{+}$. Chin Phys Lett, 2011, 28: 013201

21 Guan H, Liu Q, Huang Y, et al. A $729 \mathrm{~nm}$ laser with ultra-narrow linewidth for probing ${ }^{4} \mathrm{~S}_{1 / 2}{ }^{3} \mathrm{D}_{5 / 2}$ clock transition of ${ }^{40} \mathrm{Ca}^{+}$. Opt Commum, 2011, 284: 217-221

22 Dehmelt H. Mono-ion oscillator as potential ultimate laser frequency standard. IEEE Trans Instrum Meas, 1982, 31: 83-87

23 Drever R W P, Hall J L, Kowalski F V. Laser phase and frequency stabilization using an optical resonator. Appl Phys B, 1983, 31: 97-105

24 Guan H, Guo B, Huang G L, et al. Stabilization of $397 \mathrm{~nm}$ and 866 $\mathrm{nm}$ external cavity diode lasers for cooling a single calcium ion. Opt Commum, 2007, 274: 182-186

25 Qu W, Huang Y, Guan H, et al. $397 \mathrm{~nm}$ semiconductor laser stabilized with scanning transfer cavity. Chin J Lasers, 2011, 38: 0803008

26 Udem T, Holzwarth R, Hansch T W, et al. Optical frequency metrology. Nature, 2002, 416: 233-237

27 Cundiff S T, Ye J. Femtosecond optical frequency combs. Rev Mod Phys, 2003, 75: 325-342

28 Barwood G, Gao K, Gill P, et al. Development of optical frequency standards based upon the ${ }^{2} \mathrm{~S}_{1 / 2}{ }^{2} \mathrm{D}_{5 / 2}$ transition in ${ }^{88} \mathrm{Sr}^{+}$and ${ }^{87} \mathrm{Sr}^{+}$. IEEE 
Trans Instrum Meas, 2001, 50: 543-547

29 Bernard J E, Madej A A, Marmet L, et al. Cs-based frequency measurement of a single, trapped ion transition in the visible region of the spectrum. Phys Rev Lett, 1999, 82: 3228-3231

30 Madej A A, Bernard J E, Dubé P, et al. Absolute frequency of the ${ }^{88} \mathrm{Sr}^{+} 5 \mathrm{~s}^{2} \mathrm{~S}_{1 / 2}-4 \mathrm{~d}^{2} \mathrm{D}_{5 / 2}$ reference transition at $445 \mathrm{THz}$ and evaluation of systematic shifts. Phys Rev A, 2004, 70: 012507

31 Itano W M. External-field shifts of the $\mathrm{Hg}$ optical frequency standard. J Res NIST, 2000, 105: 829-837

32 Huang Y, Liu Q, Cao J, et al. Evaluation of the systematic shifts of a single ${ }^{40} \mathrm{Ca}^{+}$ion frequency standard. Phys Rev A, 2011, 84: 053841

33 Huang Y, Cao J, Liu P, et al. Hertz-level measurement of the ${ }^{40} \mathrm{Ca}^{+} 4 \mathrm{~s}$
${ }^{2} \mathrm{~S}_{1 / 2}-3 \mathrm{~d}{ }^{2} \mathrm{D}_{5 / 2}$ clock transition frequency with respect to the SI second through GPS. Phys Rev A, 2012, 85: 030503

34 Berkeland D J, Miller J D, Bergquist J C, et al. Minimization of ion mocromotion in a Paul trap. J Appl Phys, 1998, 83: 5025-5033

35 Safronova M S, Safronova U I. Blackbody radiation shift, multipole polarizabilities, oscillator strengths, lifetimes, hyperfine constants, and excitation energies in $\mathrm{Ca}^{+}$. Phys Rev A, 2011, 83: 012503

36 Bureau International des Poids et Mesures (BIPM). Circular T, May \& June 2010, http://www1.bipm.org/en/scientific/tai/time_ftp.html

37 Matsubara K, Li Y, Nagano S, et al. Absolute frequency measurement of the ${ }^{40} \mathrm{Ca}^{+}$clock transition using a LD-based clock laser and UTC(NICT). IEEE Int Freq Contr Symp, 2009, 1 \& 2: 751-755

Open Access This article is distributed under the terms of the Creative Commons Attribution License which permits any use, distribution, and reproduction in any medium, provided the original author(s) and source are credited. 